\title{
Editorial
}

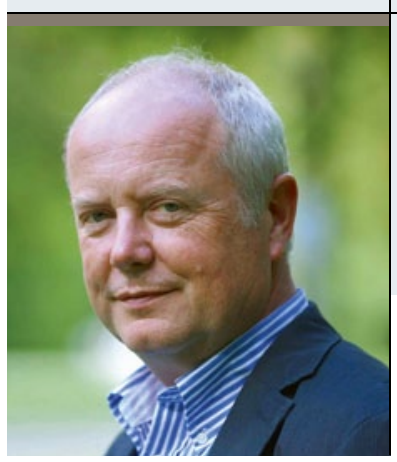

"Die Kinder- und Jugendpsychiatrie, Psychosomatik und Psychotherapie übernimmt im Netz verschiedenster Institutionen eine "Mittlerfunktion", aber auch beim Übergang in das Erwachsenenalter."

Professor Dr. med. Frank Häßler

Direktor der Klinik für Psychiatrie, Neurologie, Psychosomatik und Psychotherapie im Kindes- und Jugendalter, Rostock

\section{Gelingende Übergänge}

$\mathrm{D}$ er XXXIII. Kongress der Deutschen Gesellschaft für Kinder- und Jugendpsychiatrie, Psychosomatik und Psychotherapie, der vom 6. bis 9.3.2013 in Rostock stattfand, stand unter dem wissenschaftlichen Motto „Transition - gelingende Übergänge für psychisch und neurologisch kranke Kinder und Jugendliche". Verschiedene entwicklungsabhängige, störungsspezifische, versorgungsrelevante und vor allem disziplinenübergreifende Aspekte wurden dabei aus den unterschiedlichsten Blickwinkeln beleuchtet. Zu einigen ausgewählten Themen lesen Sie einen Kongressbericht ab Seite 16. Ein besonderes Anliegen des Kongresses war es, die Verwicklungen des Faches und seiner Repräsentanten in die Verbrechen während der NS-Zeit bewusst zu machen, die Verantwortung dafür zu übernehmen und sich bei den Opfern zu entschuldigen [1].

Vor dem Hintergrund, dass trotz demografischen Wandels die Nachfrage von Leistungen der Kinderund Jugendpsychiatrie enorm gestiegen ist [2], galt mit dem wissenschaftlichen Schwerpunktthema „Transition“ das Hauptaugenmerk allen Arten von Übergängen, die das Fachgebiet so interessant machen und die "Mittlerfunktion" der Kinder- und Jugendpsychiatrie, Psychosomatik und Psychotherapie im Netz verschiedenster Institutionen, aber auch beim Übergang in das Erwachsenenalter, verdeutlichen. Transitionen lassen sich grob in Übergänge in der Zeit (längsschnittlich) und solche zwischen sozialen Räumen oder Kategorien, Funktionen und Diagnosen (querschnittlich) teilen. Übergänge in der Zeit betreffen immer die Entwicklung unserer Patienten [3].

\section{Transition im Längsschnitt}

Die tradierte Bedeutung des Begriffes „Transition“ in der Medizin bezieht sich auf spezielle Anforderungen an die medizinische Betreuung und Versorgung im Übergang vom Kindes- und Jugendalter zum Erwachsenenalter. Potenziell betroffen ist jedes 10. Kind in Deutschland, welches gesundheitliche
Probleme aufweist, die zu entsprechenden Beeinträchtigungen des Wohlbefindens führen und damit einer dezidierten kinder- und jugendpsychiatrischen Diagnostik und Behandlung bedürfen.

Laut Richtlinien der Bundesärztekammer (1992) umfasst das medizinische Fach Kinder- und Jugendpsychiatrie die Erkennung, nicht-operative Behandlung, Prävention und Rehabilitation bei psychischen, psychosomatischen, entwicklungsbedingten und neurologischen Erkrankungen oder Störungen sowie bei psychischen und sozialen Verhaltensauffälligkeiten im Kindes- und Jugendalter, also vom Säuglingsalter bis zur Vollendung des 18. Lebensjahres (in der gerichtlichen Psychiatrie/Forensik laut Jugendgerichtsgesetz bis zur Vollendung des 21. Lebensjahres). Aus dieser Definition leitet sich die umfassende vernetzte ambulante, teilstationäre und stationäre Versorgung der betroffenen Säuglinge, Kleinkinder, Kinder, Jugendlichen und ihrer Familienangehörigen mittels vorbeugender Gesundheitspflege, Diagnostik, Beratung, Behandlung, Rehabilitation und Begutachtung ab. Dieser Aufgabe widmen sich in Deutschland rund 750 stationär und etwa 850 ambulant tätige Kinder- und Jugendpsychiater und ärztliche Psychotherapeuten. Schwierigkeiten treten aber bei der Überleitung von der psychiatrischen/psychotherapeutischen Kinder- zur psychiatrischen/psychotherapeutischen Erwachsenenmedizin auf. Diese bestehen zum einen auf der Patientenebene, da für viele Jugendliche der Wechsel vom vertrauten Kinder- und Jugendpsychiater und von seinem Behandlungsteam zum unbekannten Psychiater, der sich anderer Behandlungs- und Betreuungskonzepte bedient und oftmals auch eine ,andere Sprache" spricht, schwierig ist. Hinzu kommen altersspezifische Probleme der jungen, ,autonomen“ Erwachsenen: Sie befinden sich in der „Abnabelungsphase" und orientieren sich beruflich und privat im Leben neu. Doch viele psychische Störungen und Erkrankungen bedürfen einer kontinuierlichen medizinischen, psychopharmakologischen und/oder 
psychotherapeutischen Behandlung. Erst recht vor dem Hintergrund neuer Herausforderungen wie es der Einstieg in das Berufsleben, die Gründung einer Familie und/oder das Elternwerden mit sich bringen. Doch nicht nur aus der Versorgungsperspektive, sondern auch aus der Forschungsperspektive bedarf es vielmehr einer Reorientierung auf längsschnittliche Betrachtungen, um Frühsymptome rechtzeitig zu erkennen, einzuordnen und effektiv therapieren zu können, um Chronifizierungen vorbeugen zu können, um entwicklungspsychopathologischen Veränderungen mittels kollaborativer Mehrebenenforschung unter Einbeziehung von Genetik, Bildgebung, Neurobiologie, Neurochemie und Neurophysiologie auf den Grund zu gehen und um entwicklungsassoziierte Risiko- und Resilienzfaktoren besser zu verstehen. Unter diesem Blickwinkel wurden exemplarisch einige Krankheiten, Störungen und Syndrome in Rostock mehr als zuvor in den Fokus gerückt, wie geistige Behinderung und komorbide psychische Auffälligkeiten, Fragiles-X-Syndrom, fetales Alkoholsyndrom, frühe degenerative Erkrankungen, Säuglinge und Kleinkinder mit Regulationsstörungen oder anderen psychischen Störungen, die mit ihren Bezugspersonen auf zu schaffende Eltern-Kind-Stationen aufgenommen werden müssen, Kinder und Jugendliche psychisch kranker Eltern, Kinder und Jugendliche mit psychosomatischen Störungen, minderjährige Suchtkranke mit substanz- und nicht substanzgebundenen Abhängigkeiten, traumatisierte Kinder und nicht zuletzt sexuell übergriffige sowie delinquente Kinder und Jugendliche. Als beispielgebend und sehr effizient hat sich ein fächerübergreifendes tagesklinisches Konzept in Rostock herausgestellt. Während psychisch kranke Eltern im Rahmen der Psychiatrie tagesklinisch behandelt werden, erhalten deren ebenfalls psychisch kranke Kinder zur gleichen Zeit unter dem gleichen Dach eine entsprechende tagesklinische kinder- und jugendpsychiatrische Therapie. Bei der Umsetzung dieses Modells bedarf es einer kontinuierlichen sehr engen Absprache zwischen den jeweiligen Therapeuten und Betreuern beider Disziplinen.

\section{Transition im Querschnitt}

Transition im Querschnitt bedeutet vor allem Interdisziplinarität, um Übergänge aus und in Nachbardisziplinen sowie aus und in andere Hilfesysteme effizienter, ohne belastende, zeitraubende Doppeldiagnostik und ohne Informationsverluste zu gestalten. Um dieser Aufgabe gerecht werden zu können, kooperiert die Kinder- und Jugendpsychiatrie und -psychotherapie nicht nur mit den medizinischen Nachbarfächern wie Psychiatrie, Kinderheilkunde und Neurologie sondern auch mit der Psychologie, der Pädagogik, speziell der Sonder- und Frühpädagogik, der Justiz, der Jugend- und Sozialhilfe, dem öffentlichen Gesundheitswesen und nicht zuletzt mit der Arbeitsagentur. Diese fächerübergreifende Zusammenarbeit ist nahezu essentiell, da somatische Erkrankungen sehr häufig mit psychischen Symptomen und Störungen und damit sozialen Funktionsbeeinträchtigungen einhergehen und somati- sche Symptome wiederum sehr häufig koinzident bei psychischen Störungen auftreten. Da das Wohl des betroffenen Kindes und Jugendlichen und dessen gesunde Entwicklung der Leitgedanke allen kinder- und jugendpsychiatrischen Handelns ist, ergeben sich daraus vielfältige Aufgaben und Verpflichtungen. Vordergründig kommt es nicht auf lokal funktionierende Modellversuche oder einzelne klinische Studien an, sondern um evaluierte, flächendeckende und einfach zu praktizierende Bausteine von Prävention und Intervention. Im Sinne von evidenzbasierter Medizin gehören auch die Qualitätsbeurteilung, die Qualitätssicherung und die Qualitätsverbesserung mittels Erarbeitung von Qualitätsstandards in entsprechenden Leitlinien und deren Implementierung in der Praxis dazu. Eine Herausforderung stellt dabei die Einführung des neuen durchgängigen, leistungsorientierten und pauschalierenden Vergütungssystems auf der Grundlage von tagesbezogenen Entgelten dar. Die komplementäre bedarfsgerechte, psychiatrisch-psychotherapeutische und psychosoziale Versorgung muss sich am Patienten (dessen Bedarf sowie dessen Wohl) und dessen Lebenswelt orientieren, d.h. unter dem Motto Transition müssen zwingend die Übergänge vom ambulanten, auch aufsuchenden, über den teilstationären bis hin zum vollstationären Versorgungssektor und umgekehrt betrachtet werden. Unter dem Aspekt der „Querschnittstransition“ sollte auch die Forschung gesehen werden. Die Forschung in der Kinder- und Jugendpsychiatrie muss sich neben der Aufgeschlossenheit gegenüber neuen Trends und Forschungsansätzen in den Nachbardisziplinen Psychiatrie, Neurologie und Pädiatrie speziell der Neuropädiatrie und der Sozialpädiatrie sowohl inhaltlich als auch methodisch mehr an den patientenseitigen Erfordernissen orientieren. Wichtig und zukunftssichernd ist die Betonung einer fachspezifischen, entwicklungsorientierten Methodenkompetenz, die zu einer gewissen unverwechselbaren kinder- und jugendpsychiatrischen Forschungsidentität führt. Kein anderes neuropsychiatrisches Fach ist so prädestiniert, Ursachen, Vorläufersymptome und Verläufe psychischer Störungen mit und ohne therapeutische Interventionen vom frühesten Kindesalter bis hinein in das Erwachsenenalter zu erforschen. Die Kombination von Mehrebenenmodell im Querschnitt mit einem prospektiven Ansatz, den es gemeinsam mit der Psychiatrie zu realisieren gilt, wird entsprechend völlig neue Erkenntnisse liefern.

\section{Mit kollegialen Grüßen, Ihr}

Frank Häßler

Prof. Dr. med. Frank Häßler

Klinik für Psychiatrie, Neurologie, Psychosomatik und Psychotherapie im Kindes- und Jugendalter, Rostock E-Mail: frank.haessler@med.uni-rostock.de

Literatur unter www.springermedizin.de/dnp 